PROCEEDINGS OF THE

AMERICAN MATHEMATICAL SOCIETY

Volume 133, Number 1, Pages 229-238

S 0002-9939(04)07555-0

Article electronically published on July 26, 2004

\title{
BOUNDED AND COMPLETELY BOUNDED LOCAL DERIVATIONS FROM CERTAIN COMMUTATIVE SEMISIMPLE BANACH ALGEBRAS
}

\author{
EBRAHIM SAMEI \\ (Communicated by N. Tomczak-Jaegermann)
}

\begin{abstract}
We show that for a locally compact group $G$, every completely bounded local derivation from the Fourier algebra $A(G)$ into a symmetric operator $A(G)$-module or the operator dual of an essential $A(G)$-bimodule is a derivation. Moreover, for amenable $G$ we show that the result is true for all operator $A(G)$-bimodules. In particular, we derive a new proof to a result of N. Spronk that $A(G)$ is always operator weakly amenable.
\end{abstract}

The concept of local derivations was introduced by R. V. Kadison in [10]. An operator $D$ from a Banach algebra $A$ into a Banach $A$-bimodule $X$ is a local derivation if for each $a \in A$ there is a derivation $D_{a}$ from $A$ into $X$ such that $D(a)=D_{a}(a)$. Kadison showed that if $A$ is a von Neumann algebra and $X$ is a dual Banach bimodule, then all bounded local derivations from $A$ into $X$ are derivations. B. E. Johnson in 9] extended Kadison's result and showed that every local derivation from a $C^{*}$-algebra $A$ into any Banach $A$-bimodule is a derivation. He showed that it is enough to establish the result for the commutative regular Banach algebra $C_{0}(\mathbb{R})$. For $C_{0}(\mathbb{R})$, he first studied "local operators" and "local multipliers" from this algebra and then deduced results about local derivations. However, $C_{0}(\mathbb{R})$ is very well-behaved; it is a commutative $C^{*}$-algebra so that it is amenable and all the derivations from it into any Banach $C_{0}(\mathbb{R})$-bimodule (including those $D_{a}$ considered above) are automatically continuous. In this paper, we exploit Johnson's approach and investigate local derivations from another family of commutative regular Banach algebras which do not necessarily have the above properties. To compensate for this, we look more into the "local structure" of these algebras, and we show that most of the bounded local operators and all of the bounded local multipliers from these algebras are multipliers (sections 2 and 3 ). This provides us with the necessary tools to establish our main results on the bounded local derivations (section 4).

In section 5 , we apply the results we have obtained in the previous sections to the Fourier algebra $A(G)$ of a locally compact group $G$. Since $A(G)$ is the predual of the von Neumann algebra $V N(G)$, it has a natural operator space structure [4]. We show that every completely bounded local derivation from $A(G)$ into the

Received by the editors June 13, 2003 and, in revised form, September 30, 2003.

2000 Mathematics Subject Classification. Primary 46L07, 47B47.

Key words and phrases. Local derivations, Fourier algebra, operator space, operator weak amenability. 
operator dual of an essential $A(G)$-module is a derivation. Moreover, for amenable $G$ we show that the above result is true for all operator $A(G)$-bimodules.

Recently, N. Spronk [16] has shown that for any locally compact group $G, A(G)$ is operator weakly amenable. We give an alternative proof to Spronk's result and then prove a stronger statement that every completely bounded local derivation from $A(G)$ into any symmetric operator $A(G)$-module is zero (Proposition 4.1 and Corollary 4.4; see also Theorem 5.2(i)).

For more results on local derivations on the other families of operator algebras see [2] and [11].

\section{Preliminaries}

We have followed [3] in using some of the basic terminology not defined here. Let $A$ be a commutative regular semisimple Banach algebra with the carrier space $\Phi$. Throughout this paper we always regard $A$ as a function algebra on its carrier space. Let $F$ be a closed subset of $\Phi$. Then $F$ is a set of synthesis for $A$ if there is a unique closed ideal in $A$ whose hull is $F$. Put

$$
J_{F}(A)=\{t \in A \mid t=0 \text { on } F\}
$$

and

$$
J_{F}^{0}(A)=\{t \in A \mid t \text { has a compact support disjoint from } F\} .
$$

Then $J_{F}(A)$ is the largest and $J_{F}^{0}(A)$ is the smallest ideal in $A$ whose hull is $F$ (see, for example, [13 25D. Theorem]). So, $F$ is a set of synthesis for $A$ if and only if $J_{F}^{0}(A)$ is dense in $J_{F}(A)$.

Let $V(A)=A \otimes^{\gamma} A$ be the Banach space projective tensor product of $A$. Then $V(A)$ is a commutative regular Banach algebra with the carrier space $\Phi \times \Phi[1]$ Proposition 42.19 and Corollary 23.9]. If, in addition, $V(A)$ is semisimple (this happens, for example, when $A$ satisfies the Grothendieck approximation property [18]), then we can consider the synthesis problem for the closed subsets of $\Phi \times \Phi$. In particular, the diagonal, that is $\Delta=\{(\varphi, \varphi) \mid \varphi \in \Phi\}$, is a set of synthesis for $V(A)$ if and only if $J_{\Delta}^{0}(V(A))$ is dense in $J_{\Delta}(V(A))$. Let $\pi$ be the multiplication operator from $V(A)$ into $A$ specified by $\pi(a \otimes b)=a b$. Then, with the assumption of the semisimplicity of $V(A)$, it is easy to verify that $J_{\Delta}(V(A))=\operatorname{ker} \pi$.

Let $G$ be a locally compact group with a fixed left Haar measure. Given a function $f$ on $G$ the left translation of $f$ by $x \in G$ is denoted by $\left(l_{x} f\right)(y)=f(x y)$. Let $P(G)$ be the set of all continuous positive definite functions on $G$, and let $B(G)$ be its linear span. The space $B(G)$ can be identified with the dual of the group $C^{*}$-algebra $C^{*}(G)$, this latter being the completion of $L^{1}(G)$ under its largest $C^{*}$ norm. With pointwise multiplication and the dual norm, $B(G)$ is a commutative regular semisimple Banach algebra. The Fourier algebra $A(G)$ is the closure of $B(G) \cap C_{c}(G)$ in $B(G)$. It is shown in [6] that $A(G)$ is a commutative regular semisimple Banach algebra whose carrier space is $G$. Also, if $\lambda$ is the left regular representation of $G$ on $L^{2}(G)$ then, up to isomorphism, $A(G)$ is the unique predual of $V N(G)$, the von Neumann algebra generated by the representation $\lambda$.

\section{LOCAL OPERATORS}

Let $A$ be a commutative regular semisimple Banach algebra with the carrier space $\Phi$, and let $X$ be a Banach left (right) $A$-module. For $x \in X$, the annihilator 
$\operatorname{Ann}(x)$ of $x$ is

$$
\operatorname{Ann}(x)=\{a \in A \mid a x=0(x a=0)\} .
$$

It is clearly a closed ideal in $A$, and its hull is called the support of $x$, denoted by supp $x$. In the case $X=A$ where we regard $A$ as a Banach (left or right) $A$-module on itself, the support of an element $a \in A$ will be the closure of $\{t \in \Phi \mid a(t) \neq 0\}$. An operator $T: A \longrightarrow X$ is called a local operator if $\operatorname{supp} T(a) \subseteq \operatorname{supp} a$ for all $a \in A$.

The following is a well-known result (see, for example, [13 25.C Lemma]).

Lemma 2.1. Let $A$ be a commutative regular semisimple Banach algebra with the carrier space $\Phi$. For every compact subset $K$ and closed subset $C$ of $\Phi$ with $K \cap C=\emptyset$, there is an element $a$ in $A$ such that $a=1$ on $K$ and $a=0$ on $C$.

The following lemma is the modification of [6, Proposition 4.4].

Lemma 2.2. Let $A$ be a commutative regular semisimple Banach algebra with the carrier space $\Phi$, let $X$ be a Banach left (right) A-module, and let $x \in X$. Then $t \in \operatorname{supp} x$ if and only if for every compact neighborhood $V$ of $t$, there is an element $a \in A$, with the support in $V$, such that $a x \neq 0(x a \neq 0)$.

Proof. We prove the lemma in the case of a left module. The other case can be proved similarly. Let $t \in \operatorname{supp} x$, and assume that there is a compact neighborhood $V$ of $t$ such that for every $a \in A$, with supp $a \subseteq V$, we have $a x=0$. By Lemma 2.1 there is $b \in A$ such that supp $b \subseteq V$ and $b(t) \neq 0$. Thus, for every $a \in A$, $\operatorname{supp}(a b) \subseteq V$ and so $a b x=0$. In particular, if we take $a \in A$ such that $a=1$ on $V$, then $b x=a b x=0$. So $b \in \operatorname{Ann}(x)$, and since $t \in \operatorname{supp} x, b(t)=0$, which is a contradiction. For the converse, let $t \in \Phi$ with the given property and $a \in A$ such that $a(t) \neq 0$. We will show that $a x \neq 0$. There is a compact neighborhood $V$ of $t$ and $\delta>0$ such that $|a(v)| \geq \delta>0$ for all $v \in V$. Because of the regularity of $A$ and [15, Theorem 3.6.15], there is $b \in A$ such that $a b=1$ on $V$. Let $c \in A$ be a function whose support is in $V$ such that $c x \neq 0$. Then $a b c x=c x$; therefore, $a x \neq 0$.

Definition 2.3. A commutative regular semisimple Banach algebra $A$ is called a Tauberian algebra if the elements with compact support are dense in $A$ (see [15]). We denote the set of all such elements by $A_{c}$.

We recall that a Banach left [right] $A$-module $X$ is essential if it is the closure of $A X=\operatorname{span}\{a x \mid a \in A, x \in X\}[X A=\operatorname{span}\{x a \mid a \in A, x \in X\}]$, and a Banach $A$-bimodule $X$ is essential if it is essential both as a Banach left and right $A$-module. If $A$ is a Tauberian algebra then, by Lemma 2.1, it is easy to see that $A_{c}=A_{c}^{2}$, and so $A=\overline{A^{2}}$. Hence $A$ is essential as a Banach $A$-bimodule on itself.

Theorem 2.4. Let $A$ be a Tauberian algebra such that $V(A)$ is semisimple. Suppose that the diagonal is a set of synthesis for $V(A)$, and let $X$ be an essential Banach right (left) A-module. Then every bounded local operator $T$ from $A$ into $X^{*}$ is a multiplier.

Proof. We prove the theorem in the case of a right module. The other case can be proved similarly. First consider the case $X=A$, where we are considering $A$ as a right module on itself, and let $T: A \longrightarrow A^{*}$ be a bounded local operator. Define

$$
\tilde{T}: V(A) \longrightarrow \mathbb{C} \quad \text { by } \quad \tilde{T}(a \otimes b)=\langle T(a), b\rangle \quad(a, b \in A) .
$$


Pick $a$ and $b$ in $A_{c}$ such that $\operatorname{supp} a \cap \operatorname{supp} b=\emptyset$. By Lemma 2.1, there is $e \in A$ such that $e=1$ on $\operatorname{supp} b$, so that $b=b e$. Since $T$ is a local operator, supp $b T(a) \subseteq \operatorname{supp} b \cap \operatorname{supp} T(a)=\emptyset$. So by the Tauberian theorem [6] Theorem 3.34], Ann $b T(a)=0$. That means $b T(a)=e b T(a)=0$ and hence $\tilde{T}(a \otimes b)=\langle b T(a), e\rangle=0$. So $\tilde{T}=0$ on

$$
J=\overline{\operatorname{span}}\{a \otimes b \in V(A) \mid a \text { and } b \text { have disjoint compact supports }\} .
$$

But $J$ is a closed two-sided ideal whose hull is the diagonal. So by the hypothesis, $J=J_{\Delta}(V(A))=\operatorname{ker} \pi$. Therefore, for all $a, b, c \in A, \tilde{T}(a c \otimes b)=\tilde{T}(a \otimes c b)$. Thus

$$
\langle T(c a), b\rangle=\langle T(a c), b\rangle=\langle T(a), c b\rangle=\langle T(a), b c\rangle=\langle c T(a), b\rangle .
$$

So $T$ is a multiplier.

Now we consider the general case. Let $X$ be a Banach right $A$-module and $x \in X$. Define:

$$
K_{x}: X^{*} \longrightarrow A^{*}\left\langle K_{x}\left(x^{*}\right), a\right\rangle=\left\langle x^{*}, x a\right\rangle \quad\left(a \in A, x^{*} \in X^{*}\right) .
$$

It is easy to see that $K_{x}$ is a bounded left $A$-module homomorphism. So $K_{x} \circ T$ is a bounded local operator from $A$ into $A^{*}$ and so it is a (right) multiplier. Therefore, $\left.K_{x}(T(a b))-a T(b)\right)=0$ for all $a$ and $b$ in $A$. So for each $c \in A$, we have

$$
\langle T(a b)-a T(b), x c\rangle=0 .
$$

The final result follows by the essentiality of $X$.

\section{LOCAL MULTIPLIERS}

Let $A$ be a commutative regular semisimple Banach algebra, and let $X$ be a Banach left $A$-module. An operator $T: A \longrightarrow X$ is a right multiplier if for each $a, b \in A, T(a b)=a T(b)$. A local right multiplier is an operator $T: A \longrightarrow X$ such that for each $a \in A$ there is a right multiplier $T_{a}: A \longrightarrow X$ with $T(a)=T_{a}(a)$. Similarly, we can define (local) left multipliers for Banach right $A$-modules. In any case, we have $\operatorname{Ann}(a) \subseteq \operatorname{Ann}\left(T_{a}(a)\right)=\operatorname{Ann}(T(a))$; so supp $T(a) \subseteq \operatorname{supp} a$. Thus, a local multiplier is a local operator.

Theorem 3.1. Let $A$ be a Tauberian algebra such that $V(A)$ is semisimple. Suppose that the diagonal is a set of synthesis for $V(A)$, and let $X$ be a Banach (right or left) A-module. Then every bounded local multiplier $T$ from $A$ into $X$ is a multiplier.

Proof. We prove this for the local right multipliers. The other case can be proved similarly. Since every bounded local multiplier is a bounded local operator, a similar argument to what we have made in the proof of Theorem 2.4 (by replacing $X$ with $X^{* *}$ ) shows that

$$
c T(a b)-c a T(b)=0 \quad(a, b, c \in A) .
$$

Take $a \in A_{c}$ and $c \in A$ such that $c=1$ on $\operatorname{supp} a$. So $c a=a$. Since $\mathrm{T}$ is a local multiplier, there is a right multiplier $M$ from $A$ into $X$ such that $T(a b)=M(a b)$. Hence,

$$
0=c T(a b)-c a T(b)=c M(a b)-a T(b)=M(c a b)-a T(b)=T(a b)-a T(b) .
$$

The final result follows by the density of $A_{c}$ in $A$. 


\section{LOCAL DERIVATIONS}

Throughout this section, $A$ is a commutative regular semisimple Banach algebra with the carrier space $\Phi$. Let $X$ be a Banach $A$-bimodule. An operator $D$ from $A$ into $X$ is called a local derivation if for each $a \in A$, there is a derivation $D_{a}$ from $A$ into $X$ such that $D(a)=D_{a}(a)$.

We recall that a Banach $A$-bimodule $X$ is called a symmetric Banach $A$-module if for all $a \in A$ and $x \in X, a x=x a$. We say that $A$ is weakly amenable if every bounded derivation from $A$ into $A^{*}$ is zero, or equivalently, every bounded derivation from $A$ into any symmetric Banach $A$-module is zero [3] Theorem 2.8.63].

Proposition 4.1. Let $A$ be a Tauberian algebra such that $V(A)$ is semisimple and the diagonal is a set of synthesis for $V(A)$. Then $A$ is weakly amenable.

Proof. Let $D: A \longrightarrow A^{*}$ be a bounded derivation. Define the bounded operator $\tilde{D}: V(A) \longrightarrow \mathbb{C}$ specified by

$$
\tilde{D}(a \otimes b)=\langle D(a), b\rangle \quad(a, b \in A) .
$$

Take $a$ and $b$ in $A_{c}$ such that $a$ and $b$ have disjoint (compact) supports. By Lemma 2.1, there is an $e$ in $A$ such that $e=1$ on supp $a$ and $e=0$ on $\operatorname{supp} b$. So $e a=a$ and $e b=0$. Hence

$$
\begin{aligned}
\langle D(a), b\rangle & =\langle D(e a), b\rangle=\langle D(e) a+e D(a), b\rangle \\
& =\langle D(e), a b\rangle+\langle D(a), b e\rangle=0 .
\end{aligned}
$$

Therefore, by a similar argument to the one made in the proof of Theorem $2.4 \mathrm{D}$ is a multiplier. So for all $a, c \in A$ we have $a D(c)=D(a c)=a D(c)+D(a) c$. Thus $D(a) c=0$. Hence $D(a)=0$, since $\overline{A^{2}}=A$ by the remark made after the Definition 2.3. So $D$ is zero.

For any subset $E$ of $\Phi$ we let

$$
K(E)=\{a \in A \mid a=0 \text { on } E\},
$$

and for any two subsets $I_{1}$ and $I_{2}$ we let $V_{0}\left(I_{1}, I_{2}\right)$ be the closed linear span in $V(A)$ of the elements $a_{1} \otimes a_{2}$ where $a_{i} \in K\left(\Phi \backslash I_{i}\right), i=1,2$. It is easy to check that $V_{0}\left(I_{1}, I_{2}\right)$ is a Banach $A$-submodule of $V(A)$. The following lemma is a modification of [9, Lemma 5.2]. We include the proof for the sake of completeness.

Lemma 4.2. Let $I_{1}$ and $I_{2}$ be subsets of $\Phi$ and $\theta \in V(A)^{*}$.

(i) If $a \in K\left(I_{1}\right)$, then $\theta a \in V_{0}\left(I_{1}, I_{2}\right)^{\perp}$.

(ii) If $a \in K\left(I_{2}\right)$, then $a \theta \in V_{0}\left(I_{1}, I_{2}\right)^{\perp}$.

(iii) If $a \in A$ and $a=1$ on $I_{1}$, then $\theta-\theta a \in V_{0}\left(I_{1}, I_{2}\right)^{\perp}$.

(iv) If $a \in A$ and $a=1$ on $I_{2}$, then $\theta-a \theta \in V_{0}\left(I_{1}, I_{2}\right)^{\perp}$.

Proof. Let $c_{i} \in K\left(\Phi \backslash I_{i}\right), i=1,2$. For (i) we have $\left\langle\theta a, c_{1} \otimes c_{2}\right\rangle=\left\langle\theta, a c_{1} \otimes c_{2}\right\rangle=0$. For (iii) we have $\left\langle\theta-\theta a, c_{1} \otimes c_{2}\right\rangle=\left\langle\theta,\left(c_{1}-a c_{1}\right) \otimes c_{2}\right\rangle=0$ because $c_{1}=a c_{1}$. The other two statements can be proved similarly.

Theorem 4.3. Let $A$ be a Tauberian algebra such that $V(A)$ is semisimple. Suppose that the diagonal is a set of synthesis for $V(A)$, and let $X$ be an essential Banach A-bimodule. Then every bounded local derivation $D$ from $A$ into $X^{*}$ is a derivation. Moreover, if $A$ has a bounded approximate identity, then the statement of the theorem is true for all Banach A-bimodules. 
Proof. Consider first the case $X=V(A)$. Let $D: A \longrightarrow V(A)^{*}$ be a bounded local derivation, $I_{1}$ and $I_{2}$ be disjoint compact subsets of $\Phi$ and

$$
q: V(A)^{*} \longrightarrow V(A)^{*} / V_{0}\left(I_{1}, I_{2}\right)^{\perp}
$$

be the natural quotient map. Put $\tilde{D}=q \circ D$. Since $q$ is a bounded $A$-module homomorphism, $\tilde{D}$ is a bounded local derivation. Now, let $b_{1} \in K\left(I_{1}\right)$ and define

$$
T_{1}: A \longrightarrow V(A)^{*} / V_{0}\left(I_{1}, I_{2}\right)^{\perp} T_{1}(a)=\tilde{D}\left(a b_{1}\right) \quad(a \in A) .
$$

Since $\tilde{D}$ is a local derivation, for each $a \in A$, there is a derivation $S: A \longrightarrow$ $V(A)^{*} / V_{0}\left(I_{1}, I_{2}\right)^{\perp}$ such that $\tilde{D}\left(a b_{1}\right)=S\left(a b_{1}\right)$. So, by Lemma 4.2 (i), we have

$$
T_{1}(a)=S\left(a b_{1}\right)=a S\left(b_{1}\right)+S(a) b_{1}=a S\left(b_{1}\right) .
$$

Thus, $T_{1}$ is a bounded local right multiplier, and so it is a right multiplier, by Theorem 3.1. Hence for all $a, c \in A$,

$$
\tilde{D}\left(a c b_{1}\right)=a \tilde{D}\left(c b_{1}\right) .
$$

Similarly, we can show that for all $a, c \in A$ and $b_{2} \in K\left(I_{2}\right)$,

$$
\tilde{D}\left(a c b_{2}\right)=\tilde{D}\left(c b_{2}\right) a .
$$

Let $a, c \in A_{c}$, and let $U$ be a compact neighborhood in $\Phi$ such that $I_{2} \subseteq U$ and $U \cap I_{1}=\emptyset$. By Lemma 2.1, there are $b, e$ and $b_{1}$ in $A$ such that $b=1$ on $\operatorname{supp} a \cup \operatorname{supp} c \cup U \cup I_{1}, e=1$ on $I_{2}$ and $e=0$ outside of $U$, and finally $b_{1}=0$ on $I_{1}$ and $b_{1}=1$ on $I_{2}$. Put $b_{2}=b-b_{1}$. Then

$$
b_{i} \in K\left(I_{i}\right), a b=a, b c=c \text {, and } e b=e .
$$

Since $\tilde{D}$ is a local derivation, there is a derivation $\Delta$ from $A$ into $V(A)^{*} / V_{0}\left(I_{1}, I_{2}\right)^{\perp}$ such that $\tilde{D}\left(b^{2}\right)=\Delta\left(b^{2}\right)$. So by Lemma 4.2 (iii) and (iv), $\tilde{D}\left(b^{2}\right)=\Delta\left(b^{2}\right)=$ $e \Delta\left(b^{2}\right)=\Delta\left(e b^{2}\right)-\Delta(e) b^{2}=\Delta(e)-\Delta(e)=0$. Thus $\tilde{D}\left(b^{2}\right)=0$. On the other hand, from (4.1), (4.2) and (4.3) we have

$$
\tilde{D}(a)=\tilde{D}\left(a b^{2}\right)=\tilde{D}\left(a b\left(b_{1}+b_{2}\right)\right)=\tilde{D}\left(a b b_{1}\right)+\tilde{D}\left(a b b_{2}\right)=a \tilde{D}\left(b b_{1}\right)+\tilde{D}\left(b b_{2}\right) a .
$$

But $\tilde{D}\left(b b_{1}\right)+\tilde{D}\left(b b_{2}\right)=\tilde{D}\left(b^{2}\right)=0$. So, if we put $\theta=\tilde{D}\left(b b_{1}\right)$, then

$$
\tilde{D}(a)=a \theta-\theta a .
$$

Similarly, we can show that (4.4) holds with the same $\theta$ if we replace $a$ by either $c$ or $a c$. Therefore, $\tilde{D}(a c)=a \tilde{D}(c)+\tilde{D}(a) c$. Since $a$ and $c$ were arbitrary elements in $A_{c}$, by the density, we can conclude that $\tilde{D}$ is a derivation into $V(A)^{*} / V_{0}\left(I_{1}, I_{2}\right)^{\perp}$.

Consider $\delta D$ given by $\delta D(a, b)=a D(b)-D(a b)+D(a) b$. It is a 2-cocycle from $A$ with values in $V(A)^{*}$. However, because $\tilde{D}$ is a derivation, $\delta D$ maps into $V_{0}\left(I_{1}, I_{2}\right)^{\perp}$, and since this holds for all the choices of $I_{1}$ and $I_{2}, \delta D$ maps into

$$
\left(\overline{\operatorname{span}}\left\{V_{0}\left(I_{1}, I_{2}\right) \mid I_{1} \text { and } I_{2} \text { are disjoint compact sets }\right\}\right)^{\perp},
$$

which is $J_{\Delta}(V(A))^{\perp}$, by the assumption that $\Delta$, the diagonal, is a set of synthesis for $V(A)$. On the other hand, $J_{\Delta}(V(A))^{\perp}=(\operatorname{ker} \pi)^{\perp} \cong(V(A) / \operatorname{ker} \pi)^{*}$. So $\delta D$ maps into $(V(A) / \operatorname{ker} \pi)^{*}$, which is the dual of a symmetric essential Banach $A$-module. Fix $b \in A$ and define a bounded operator $\mathcal{D}: A \longrightarrow(V(A) / \operatorname{ker} \pi)^{*}$ by

$$
\mathcal{D}(a)=\delta D(a, b) \quad(a \in A) .
$$

We claim that $\mathcal{D}$ is a local operator. Let $a \in A$ and $t \notin \operatorname{supp} a$. So there is a compact neighborhood $V$ of $t$ such that supp $a \cap V=\emptyset$. Take $c \in A$ with 
supp $c \subseteq V$. By Lemma 2.1, there is $e \in A$ such that $e=1$ on $V$ and $e=0$ on supp $a$. Then, since $V(A) / \operatorname{ker} \pi$ is symmetric and $c a=0$,

$$
c \mathcal{D}(a)=c e \mathcal{D}(a)=c \delta D(a, b) e=-c D(a b) e+c D(b) e b .
$$

On the other hand, let $h \in A$ be any element such that $c h=e h=0$, and let $\Delta: A \longrightarrow V(A)^{*}$ be a derivation such that $D(h)=\Delta(h)$. Then $c D(h) e=c \Delta(h) e=$ $\Delta(c h) e-\Delta(c) h e=0$. Thus, from (4.5), $c \mathcal{D}(a)=0$, and so by Lemma [2.2, $t \notin$ $\operatorname{supp} \mathcal{D}(a)$, i.e., $\mathcal{D}$ is a bounded local operator; so by Lemma 2.4, it is a multiplier. Hence, for all $a, b, c \in A, \delta D(a c, b)=a \delta D(c, b)$. So

$$
D(a c b)-D(a c) b=a D(c b)-a D(c) b .
$$

Now, take $a, b \in A_{c}$ and $c \in A$ such that $c=1$ on supp $a \cup \operatorname{supp} b$. Then from (4.6),

$$
D(a b)-D(a) b-a D(b)=-a D(c) b .
$$

However, $D$ is a local derivation; so, there is a derivation $N$ from $A$ into $V(A)^{*}$ such that $D(c)=N(c)$. So

$$
a D(c) b=a N(c) b=N(a c) b-N(a) c b=N(a) b-N(a) b=0 .
$$

Hence, from (4.7), $\delta D(a, b)=0$ for all $a, b \in A_{c}$. Therefore, by the density, $\delta D=0$ and so $D$ is a derivation.

We now consider the general case. Let $x \in X$, and define $L_{x}: X^{*} \longrightarrow V(A)^{*}$ by

$$
\left\langle L_{x}\left(x^{*}\right), a \otimes b\right\rangle=\left\langle x^{*}, a x b\right\rangle \quad\left(a, b \in A, x^{*} \in X^{*}\right) .
$$

It is easy to check that $L_{x}$ is a bounded $A$-bimodule homomorphism, and hence $L_{x} \circ D$ is a bounded local derivation into $V(A)^{*}$. Thus $L_{x}(\delta D(c, e))=0$ for all $c, e \in A$ and $x \in X$. So $\langle\delta D(c, e), a x b\rangle=0$ for all $a, b \in A$ and $x \in X$. Thus, by the essentiality of $X, \delta D=0$, showing that $D$ is a derivation.

Finally, suppose that $A$ has a bounded approximate identity, $X$ is a Banach $A$ bimodule and $D: A \longrightarrow X$ is a bounded local derivation. By a similar argument to the one made above (by replacing $X$ with $X^{* *}$ ), we can show that for all $a, b, c, d \in$ $A$,

$$
c \delta D(a, b) d=0 .
$$

Put $Y=X A$. By Cohen's factorization theorem [1, Theorem 11.10], $Y$ is a closed submodule of $X$. Let $q$ be the natural quotient map from $X$ onto $X / Y$. Let $\left\{e_{\alpha}\right\}_{\alpha \in \Lambda}$ be a bounded approximate identity for $A$. For each $\alpha \in \Lambda$ define $T_{\alpha}: A \longrightarrow X / Y$ by

$$
T_{\alpha}(a)=q\left(D\left(a e_{\alpha}\right)\right) \quad(a \in A) .
$$

It is easy to see that $T_{\alpha}$ is a bounded local right multiplier; so it is a right multiplier by Theorem 3.1 Hence, for all $a, b \in A$ and $\alpha \in \Lambda$, we have $q\left(D\left(a b e_{\alpha}\right)\right)=$ $a q\left(D\left(b e_{\alpha}\right)\right)$. By letting $\alpha \longrightarrow \infty$, we see that $q(D(a b)-a D(b))=0$. So $D(a b)-$ $a D(b) \in X A$. Hence $\delta D(a, b) \in X A$. So, by Cohen's factorization theorem, there is $e \in A$ and $x \in X$ such that $\delta D(a, b)=x e$. So if we put $d=e_{\alpha}$ in (4.8) and let $\alpha \longrightarrow \infty$ we will get $c \delta D(a, b)=0$. Similarly, by letting $Y=A X$, we can show that $\delta D(a, b)=0$ for all $a, b \in A$. So $D$ is a derivation.

Corollary 4.4. Let $A$ be a Tauberian algebra such that $V(A)$ is semisimple. Suppose that the diagonal is a set of synthesis for $V(A)$, and let $X$ be a symmetric Banach A-module. Then every bounded local derivation $D$ from $A$ into $X$ is zero. 
Proof. First consider the case $X=A^{*}$. Since $X$ is the dual of an essential Banach $A$ bimodule, by Theorem $4.3 D$ is a bounded derivation and therefore, by Proposition 4.1. $D=0$. For the general case, by a similar argument to the one made in the proof of Theorem 2.4 (by replacing $X$ with $X^{* *}$ ) we have

$$
b D(a)=0 \quad(a, b \in A) .
$$

Now, let $a, b \in A_{c}$ and take $c \in A$ such that $c=1$ on supp $a \cup \operatorname{supp} b$. So $a c=a$ and $b c=b$. Since $D$ is a local derivation, there is a derivation $S$ from $A$ into $X$ such that $D(a b)=S(a b)$. Hence,

$$
D(a b)=S(a b c)=a b S(c)+S(a b) c=a S(c) b+D(a b) c .
$$

But $D(a b) c=0$ by (4.1), and $a S(c) b=S(a c) b-S(a) c b=S(a) b-S(a) b=0$. So $D=0$ on $A_{c}^{2}$. Therefore, by the density, $D=0$.

Corollary 4.5. Let $G$ be a locally compact group such that it has an abelian subgroup of finite index. Then every bounded local derivation from $A(G)$ into any Banach $A(G)$-bimodule is a derivation.

Proof. By a result of Losert in [14, $A(G) \widehat{\otimes} A(G) \cong A(G \times G)$. Thus, since the diagonal $\Delta(G)$ is a closed subgroup of $G \times G$, by [17] Theorem 3] $\Delta(G)$ is a set of synthesis for $V(A(G))$. So we have the result from Theorem 4.3

\section{Completely Bounded local Derivations on the Fourier algebra}

Let $H$ be a Hilbert space, and let $B(H)$ denote the space of all bounded linear operators on $H$. For each $n \in \mathbb{N}$, there is a natural operator norm $\|\cdot\|_{n}$ on the $n \times n$ matrix space $M_{n}(B(H)) \cong B\left(H^{n}\right)$. This family of norms $\left\{\|\cdot\|_{n}\right\}$ is the operator matrix norm on $B(H)$. An operator space is a (normed closed in this paper) linear subspace of $B(H)$ together with the operator matrix norms inherited from $B(H)$. Let $V$ and $W$ be two operator spaces and $\phi: V \longrightarrow W$ a linear map. For each $n \in \mathbb{N}, \phi$ induces a linear map $\phi_{n}: M_{n}(V) \longrightarrow M_{n}(W)$ defined by

$$
\phi_{n}\left(\left[v_{i j}\right]\right)=\left[\phi\left(v_{i j}\right)\right]
$$

for $\left[v_{i j}\right] \in M_{n}(V)$. The completely bounded norm of $\phi$ is

$$
\|\phi\|_{c b}=\sup \left\{\left\|\phi_{n}\right\| \mid n \in \mathbb{N}\right\} .
$$

Then $\phi$ is completely bounded (resp. completely contractive, completely isometric) if $\|\phi\|_{c b}<\infty$ (resp. $\|\phi\|_{c b} \leq 1$, each $\phi_{n}$ is an isometry). We let $C B(V, W)$ denote the space of all completely bounded maps from $V$ into $W$. It is shown in [4 that there is a natural operator space structure on $C B(V, W)$ obtained by identifying $M_{n}(C B(V, W)) \cong C B\left(V, M_{n}(W)\right)$. Thus for every operator space $V$, its Banach dual space $V^{*}=B(V, \mathbb{C})=C B(V, \mathbb{C})$ is again an operator space and is called the operator dual of $V$. If we let $V \hat{\otimes} W$ be the operator projective tensor product of $V$ and $W$ (see [4, Chapter 7]), then there is a complete isometry $C B\left(V, W^{*}\right) \cong$ $(V \hat{\otimes} W)^{*}$ given by

$$
\langle\widetilde{T}(v), w\rangle=\langle T, v \otimes w\rangle \quad\left(v \in V, w \in W, T \in(V \hat{\otimes} W)^{*}\right) .
$$

Also, if $Z$ is an operator space, then there are natural complete isometric isomorphisms $V \hat{\otimes} W \cong W \hat{\otimes} V$ and $(V \hat{\otimes} W) \hat{\otimes} Z \cong V \hat{\otimes}(W \hat{\otimes} Z)$.

Let $A$ be a Banach algebra that is additionally an operator space. $A$ is called a completely contractive Banach algebra if the multiplication $m: A \hat{\otimes} A \longrightarrow A$ is 
completely contractive. Moreover, if $X$ is a Banach $A$-bimodule, then $X$ is called an operator A-bimodule if $X$ is an operator space and the $A$-bimodule operations

$$
A \hat{\otimes} X \longrightarrow X ; a \otimes x \longmapsto a x
$$

and

$$
X \hat{\otimes} A \longrightarrow X ; x \otimes a \longmapsto x a
$$

are completely bounded. It is easy to check that there is a natural operator $A$ bimodule structure on $X^{*}$. A is called operator weakly amenable if every completely bounded derivation from $A$ into $A^{*}$ is inner [7]. By [7] Proposition 3.2], for $A$ commutative, this is equivalent to saying that every completely bounded derivation from $A$ into any symmetric operator $A$-module is zero. The following lemma is well known and easy to check; so we omit the proof.

Lemma 5.1. Let $A$ be a completely contractive Banach algebra, let $X$ be an operator A-bimodule, and let $x \in X$. Then, the operators $K_{x}$ and $L_{x}$ defined by

$$
\begin{aligned}
& K_{x}: X^{*} \longrightarrow A^{*},\left\langle K_{x}\left(x^{*}\right), a\right\rangle=\left\langle x^{*}, x a\right\rangle \quad\left(a \in A, x^{*} \in X^{*}\right), \\
& L_{x}: X^{*} \longrightarrow(A \hat{\otimes} A)^{*},\left\langle L_{x}\left(x^{*}\right), a \otimes b\right\rangle=\left\langle x^{*}, a x b\right\rangle \quad\left(a, b \in A, x^{*} \in X^{*}\right)
\end{aligned}
$$

are completely bounded.

Let $G$ be a locally compact group. Then, the von Neumann algebra $V N(G) \subset$ $B\left(L^{2}(G)\right)$ is an operator space. Thus $A(G)$, regarded as the operator predual of $V N(G)$, has a natural operator space structure that makes it a completely contractive Banach algebra [4, Chapter 16].

Theorem 5.2. Suppose that $G$ is a locally compact group. Then

(i) Every completely bounded local derivation from $A(G)$ into any symmetric operator $A(G)$-module is zero. In particular, $A(G)$ is operator weakly amenable.

(ii) Every completely bounded local derivation from $A(G)$ into the operator dual of an essential $A(G)$-bimodule is a derivation.

(iii) If $G$ is amenable, then the statement in (ii) is true for all operator $A(G)$ bimodules.

Proof. By replacing "Banach algebra" with "completely contractive Banach algebra", "bounded" with "completely bounded", "projective tensor product" with "operator projective tensor product", "Banach module" with "operator module" and "weakly amenable" with "operator weakly amenable", we see that the quantized versions of Theorems 2.4 and 3.1, Proposition 4.1. Theorem 4.3 and Corollary 4.4 are valid (see [4 Chapters 3,4 and 7] and Lemma 5.1 for the details). On the other hand, it is shown in [5] that there is a complete isometry $A(G) \hat{\otimes} A(G) \cong A(G \times G)$. This map is also an algebraic isomorphism. So $A(G) \hat{\otimes} A(G)$ is semisimple and, since the diagonal $\Delta(G)=\{(g, g) \mid g \in G\}$ is a closed subgroup of $G \times G$, by 17 . Theorem 3] $\Delta(G)$ is a set of synthesis for $A(G) \hat{\otimes} A(G)$. Hence, by the quantized versions of Theorem 4.3 and Corollary 4.4 the statements (i)-(iii) above follow. We note that in (iii) we are using the fact that if $G$ is amenable, then $A(G)$ has a bounded approximate identity [12].

Forrest and Wood have shown in [7, Theorem 4.5] that if $G$ is a locally compact group such that it has an abelian subgroup of finite index, then every bounded linear mapping from $A(G)$ into any operator space is completely bounded. So (by some modification) Corollary 4.5 follows from Theorem 5.2 


\section{ACKNOWLEDGMENT}

The author would like to express his deep gratitude to Professor F. Ghahramani for his invaluable and constructive comments during this work. The author also would like to thank the referee for helpful comments which have improved the paper.

\section{REFERENCES}

[1] F. F. Bonsall and J. Duncan, Complete Normed Algebras, New York, Springer-Verlag 1973. MR.54:11013

[2] Randel L. Crist, Local derivations on operator algebras, Journal of Functional Analysis 135 (1996), 76-92. MR96m:46128

[3] H. G. Dales, Banach algebras and automatic continuity, New York, Oxford University Press, 2000. MR 2002e:46001

[4] E. G. Effros and Z.-J. Ruan, Operator spaces, London Math. Soc. Monograghs, New series, vol. 23, Oxford University Press, New York, 2000. MR2002a:46082

[5] E. G. Effros and Z.-J. Ruan, On approximation properties for operator spaces, Internat. J. Math. 1 (1990), 163-187. MR92g:46089

[6] P. Eymard, L'algèbre de Fourier d'un groupe localement compact, Bull. Soc. Math. France 92 (1964), 181-236. MR 37:4208

[7] B. E. Forrest and P. J. Wood, Cohomology and the operator space structure of the Fourier algebra and its second dual, Indiana Math. J. 50 (2001), 1217-1240. MR.2003d:43007

[8] C. S. Herz, Harmonic synthesis for subgroups. Ann. Inst. Fourier, Grenoble 23 (1973), no. 3, 91-123. MR50:7956

[9] B. E. Johnson, Local derivations on $C^{*}$-algebras are derivations, Trans. Amer. Math. Soc. 353 (2000), 313-325. MR2002c:46132

[10] R. V. Kadison, Local derivations, J. Algebra 130 (1990), 494-509. MR91f:46092

[11] D. Larson and A. Sourour, Local derivations and local automorphisims of $B(X)$, Proc. Sympos. Pure Math. 51 (1990), 187-194. MR91k:47106

[12] H. Leptin, Sur l'algèbre de Fourier d'un groupe localement compact, C. R. Acad. Sci. Paris Sér. A-B 266 (1968), 1180-1182. MR39:362

[13] L. H. Loomis, An introduction to abstract harmonic analysis, New York, Van Nostrand, 1953. MR:14:883c

[14] V. Losert, On tensor products of Fourier algebras, Arch. Math. 43 (1984), 370-372. MR $87 \mathrm{c}: 43004$

[15] C. E. Rickart, General theory of Banach algebra, Princeton, N.J., Van Nostrand, 1960. MR22:5903

[16] N. Spronk, Operator weak amenability of the Fourier algebra, Proc. Amer. Math. Soc. 130 (2002), 3609-3617. MR2003f:46091

[17] M. Takesaki and N. Tatsuuma, Duality and subgroups. II, J. Functional Analysis 11 (1972), 184-190. MF 52:5865

[18] J. Tomiyama, Tensor products of commutative Banach algebras, Tôhoku Math. J. (2) 12 (1960), 143-154. MR 22:5910

Department of Mathematics, University of Manitoba, Winnipeg, Manitoba, Canada R3T $2 \mathrm{~N} 2$

E-mail address: umsameie@cc.umanitoba.ca 